\title{
Calidad de vida en adultos mayores de Guerrero, México
}

\author{
Quality of life in elderly adults from Guerrero, Mexico \\ Elvia Peña-Marcial1* orcid.org/0000-0003-1232-6000 \\ Lorena Inés Bernal-Mendoza1 orcid.org/0000-0002-1799-9522 \\ Leticia Reyna-Avila ${ }^{1}$ orcid.org/0000-0001-9862-4062 \\ Rodrigo Pérez-Cabañas ${ }^{1}$ orcid.org/0000-0002-3417-2308 \\ Derly Asmir Onofre-Ocampo² orcid.org/0000-0002-7648-9645 \\ Ingrid Astrid Cruz-Arteaga ${ }^{2}$ orcid.org/0000-0002-6009-944X \\ Dulce Anel Silvestre-Bedolla² orcid.org/0000-0002-8540-2759
}

\begin{abstract}
1 Docente investigador de la Escuela Superior de Enfermería No. 1, Universidad Autónoma de Guerrero. Chilpancingo, México
2 Pasante de Licenciatura en Enfermería, Universidad Autónoma de Guerrero. Chilpancingo, México
\end{abstract}

\section{Resumen}

Introducción: La calidad de vida se define como un estado de bienestar físico, social, emocional, espiritual, intelectual y ocupacional que le permite al individuo satisfacer apropiadamente sus necesidades; un grado de adaptación a su propia condición y a su medio, en función de su estado de salud, sus carencias y del soporte que recibe para suplirlas. Objetivo: Describir la calidad de vida desde el ámbito social en el que se desarrollan los adultos mayores en el Estado de Guerrero, México. Materiales y métodos: Investigación cuantitativa de corte transversal y analítico, la muestra fue probabilística, se incluyeron 75 adultos mayores de 65 años y más, residentes de asilo, casa de día y localidad rural; el criterio de selección aplicado fue la firma previa del consentimiento informado. Se utilizó el Cuestionario sociodemográfico y Cuestionario de Salud SF-36 versión 2 española Health Survey, constituido por preguntas agrupadas con sus respectivos ítems. Resultados: El $80 \%$ de los adultos mayores encuestados manifestó mala calidad de vida. Conclusiones: Los adultos mayores guerrerenses presentan porcentajes altos de problemas físicos, emocionales, y sociales, que dan origen a una mala perspectiva de su salud y vida.

Palabras clave: Calidad de vida; adulto; percepción. (Fuente: DeCS, Bireme).

\begin{abstract}
Introduction: Quality of life is defined as a state of social, emotional, spiritual, intellectual and occupational wellbeing. Quality of life allows individuals to properly satisfy their needs and reach a level of adaptation to their own conditions and environments according to their health status, limitations and the support they receive to supplement them. Objective: To describe the quality of life of elderly adults in the State of Guerrero-Mexico, taking into account the social context where they live. Materials and methods: Quantitative research using a cross-sectional and analytical approach. The sample was probabilistic, with 75 adults older than or equal to 65 years of age, who were residents of asylums, day houses or rural areas. The applied selection criterion was their signature of the informed consent form. We used the sociodemographic questionnaire and the Spanish version 2 of the SF-36 Health Survey questionnaire that contains questions grouped within their respective items. Results: $80 \%$ of the older adults manifested a poor quality of life. Conclusions: Elderly adults from Guerrero displayed high percentages of physical, emotional and social problems, which ultimately lead to poor health and life perspectives.
\end{abstract}

Key words: Quality of life; adult; perception. (Source: DeCS, Bireme). 


\section{Introducción}

El envejecimiento se presenta como un proceso ascendente no solo de las personas que cumplen 60 años, sino también de las personas con más de 80 años(1). De 2015 a 2050 la población mundial con más de 60 años pasará de 900 millones hasta 2000 millones, representando un aumento del $12 \%$ al $22 \%(2)$.

Según Consejo Nacional de Población (CONAPO), en el país la ciudad de México es la más envejecida, donde las personas en edades avanzadas representan 13\% de la población total de México, seguida por un conjunto de estados con proporciones mayores a 10\%, como son Veracruz, Oaxaca, Morelos, Yucatán, Michoacán, Sinaloa, Zacatecas, San Luis Potosí, Nayarit, Hidalgo, Guerrero, Jalisco y Durango. No obstante, se estima que en los próximos años todas las ciudades del país avanzarán en este proceso, algunas a ritmos más acelerados que otras. Así, para 2030, la Ciudad de México será una población envejecida (20\% de su población tendrá 60 años y más), mientras que el resto de los estados se encontrarán en proceso de transición, con proporciones de adultos mayores entre $10,63 \%$ y $16,47 \%{ }^{(3)}$.

La población adulta mayor ha crecido en los últimos años y se prevé que vaya en aumento, debido a los avances de la ciencia, medicina, higiene, nutrición y a estilos de vida más favorables; sin embargo, la longevidad tiene exigencias propias que no pueden ocultarse, como el alto costo necesario para llegar a la senectud, compensado con una vida de calidad(4). La diversidad en la vejez no es casual, se debe a la herencia genética o a las decisiones tomadas por las personas durante su vida, la mayor parte son el resultado de influencias que a menudo están fuera de su control o de las opciones que tienen a su disposición, dado que los entornos físicos y sociales que las personas habitan pueden influir en la salud directamente, por medio de obstáculos o incentivos que afectan las oportunidades, las decisiones y el comportamiento(5).

Calidad de vida es un expresión lingüística cuyo significado es eminentemente subjetivo, está asociada con la personalidad el bienestar y la satisfacción por la vida que lleva el individuo, y su evidencia esta intrínsecamente relacionada con múltiples factores como su propia experiencia, su salud y su grado de interacción social y ambiental(6).
La calidad de vida se define como un estado de bienestar físico, social, emocional, espiritual, intelectual y ocupacional que le permite al individuo satisfacer apropiadamente sus necesidades(7), es decir un grado de adaptación a su propia condición y medio, en función de su estado de salud, carencias y soporte que recibe para suplirlas ${ }^{(8)}$.

La Organización Mundial de la Salud (OMS) concibe la calidad de vida como "la percepción que un individuo tiene de su lugar en la existencia, en el contexto de la cultura y del sistema de valores en los que vive y en relación con sus objetivos, sus expectativas, sus normas, sus inquietudes". Se trata de un concepto muy amplio que está influido de modo complejo por la salud física del sujeto, su estado psicológico, su nivel de independencia, sus relaciones sociales, así como su relación con los elementos esenciales de su entorno(7).

La calidad de vida del adulto mayor, es "la resultante de la interacción entre las diferentes características de la existencia humana (vivienda, vestido, alimentación, educación y libertades humanas); cada una de las cuales contribuye de diferente manera para permitir un óptimo estado de bienestar, teniendo en cuenta el proceso evolutivo del envejecimiento, las adaptaciones del individuo a su medio biológico y psicosocial cambiante, el cual se da en forma individual y diferente; adaptación que influye en su salud física, fallas en la memoria, el temor, el abandono, la muerte, la dependencia o la invalidez"(6). La calidad de vida del adulto mayor se da en la medida en que él logre reconocimiento a partir de relaciones sociales significativas; esta etapa será vivida como continuación de un proceso vital o se vivirá como una fase de declinación funcional y aislamiento social(9).

O'Shea, sostiene que "la calidad de vida del adulto mayor es una vida satisfactoria, bienestar subjetivo y psicológico, desarrollo personal y diversas representaciones de lo que constituye una buena vida, y que se debe indagar, preguntando al adulto mayor, sobre cómo da sentido a su propia vida, en el contexto cultural, y de los valores en el que vive, y en relación a sus propios objetivos de vida"(10).

El adulto mayor suele sentir que ya no es tomado en cuenta por los demás, y que los demás no cuentan con él, siendo en el nivel familiar donde poco a poco su presencia no es tan necesaria a nivel familiar, además 
del deterioro de funcionalidad y autonomía que limitan la ejecución de actividades de la vida diaria(7).

El estudio realizado en Chiclayo, Perú, en el año 2009, sobre percepción de la calidad de vida relacionada con la salud del adulto mayor residente de esa ciudad, identificó que respecto a la encuesta SF-36 las dimensiones función social (FS), salud mental (SM) y la vitalidad (VT) obtuvieron puntuaciones de 73\%, $69 \%$ y $65 \%$ respectivamente; siendo las dimensiones más bajas las relacionadas a salud general (SG), Rol físico (RF) y dolor corporal (DC) con el 50\%, 61\%, y $62 \%$, respectivamente ${ }^{(11)}$.

En Colombia, en el año 2013 el estudio "Calidad de vida de los adultos mayores de Medellín"; mostró que el 71\% se consideraron independientes para realizar actividades de la vida diaria, 45,7\% presentaba sintomatología depresiva, 33\% presentaron riesgo de ansiedad, $28,3 \%$ deterioro funcional y $54,3 \%$, posible malnutrición. La asociación fue negativa con la calidad de vida, el ser mujer, presentar diabetes, y altos puntajes en las escalas de capacidad funcional, depresión y ansiedad(12).

En Colombia, en el año 2015 la investigación titulada "Calidad de vida en adultos mayores, ancianos jóvenes y ancianos maduros institucionalizados y no institucionalizados en la ciudad de Pereira"; evidenció que los participantes no institucionalizados presentan mejor calidad de vida y estado mental que los institucionalizados, sin embargo, los adultos mayores institucionalizados estuvieron dentro del rango de 65 a 85 años mientras que la otra muestra estuvo en el rango de 55 a 85 años, presentado poca diferencia en la media y desviación estándar(13).

El estudio realizado sobre calidad de vida y estrategias de afrontamiento ante problemas y enfermedades en ancianos de la ciudad de México, encontró que $63,9 \%$ padece alguna enfermedad, principalmente crónico-degenerativa en un 53,9\%. La calidad de vida fue significativamente diferente para los enfermos en relación con los no enfermos, no así para el tipo de enfermedad, problemas reportados y nivel de afrontamiento. Los problemas más frecuentes fueron el familiar $(33,7 \%)$ y el estilo de afrontamiento orientado a la conducta $(54,2 \%)^{(14) .}$

En el estudio soledad, depresión y calidad de vida en adultos mayores mexicanos realizado en el año 2017, incluyo a 489 adultos mayores de 60 y 97 años. Los resultados mostraron que las percepciones negativas de la vejez, la percepción de soledad social y familiar, explican principalmente los síntomas depresivos y el deterioro en la calidad de vida; además se encontró que a mayor edad hay más riesgo, y la presencia de enfermedades y la ausencia de pareja también aumentan el riesgo de presentar depresión(15).

Actualmente en la sociedad existe una cierta negación a la vejez, que se traduce en desajustes y desequilibrios psicológicos individuales, es decir la falta de aceptación del hombre en su realidad, así como la insolidaridad colectiva hacia este importante sector de la población(16).

A medida que las personas envejecen, los factores sociales e individuales relacionados con el nivel de vida, son determinantes para este grupo poblacional; por ello se describe la calidad de vida desde el ámbito social en el que se desarrollan los adultos mayores en el estado de Guerrero, México. Para ello se utilizó la encuesta SF-36 que evalúa aspectos de la calidad de vida en poblaciones adultas mediante 8 dimensiones que integran: función física, rol físico, dolor corporal, salud general, vitalidad, función social, rol emocional y salud mental. La información servirá para tener insumos que permitan generar políticas públicas en este grupo poblacional.

\section{Materiales y métodos}

\section{Diseño y población del estudio}

Estudio cuantitativo de corte transversal, analítico realizado en Chilpancingo (Guerrero), en el periodo comprendido entre los meses de abril-junio 2018; la población la integraron setenta y cinco personas adultas mayores de ambos sexos, de las cuales veinticinco eran residentes de asilo, veinticinco de la casa de día y veinticinco adultos mayores que no asisten ni dependen de alguna de estas instituciones y son habitantes de zona urbana; la participación fue voluntaria con previa firma de un consentimiento informado.

\section{Instrumentos}

Los instrumentos utilizados fueron: Cuestionario de datos sociodemográficos; constituido por preguntas con información de edad, sexo, estado civil, ocupación entre otras. Cuestionario de Salud SF-36 Versión 2(17) constituido por preguntas agrupadas con sus respectivos ítems que valoran la Función física (me limita mucho, me limita un poco, no me limita nada); 
Rol físico (Si, No); Dolor corporal (nada, un poco, regular, bastante, mucho); Salud general ( $\mathrm{Si}, \mathrm{no})$; Vitalidad (siempre, casi siempre, muchas veces, algunas veces, nunca); Función social (nada, un poco regular, bastante. mucho); Rol emocional (si-no) y Salud mental (siempre, casi siempre, muchas veces, algunas veces, nunca), además incluye el concepto general de cambios en la percepción del estado de salud actual y en el año anterior (buena, regular, mala/mucho mejor, igual que hace un año, peor). La respuesta a esta pregunta describe la transición de la percepción respecto al mejoramiento o empeoramiento del estado de salud.

La técnica de recolección de la información de estudio fue por medio de la entrevista aplicada a 75 participantes con una duración aproximada de 15 minutos.

\section{Análisis estadístico}

Para la captura del análisis de la información obtenida, se creó una base de datos en el paquete estadístico PASW Statistics 21, donde se realizó un análisis estadístico descriptivo a las características de una variable a la vez, con lo que se exploró el comportamiento de calidad de vida, entre otras a través de promedios. De la misma manera, se realizó el cruce de variables previamente agrupadas entre sí, por medio de tablas de contingencia, donde se buscó la influencia de la variable independiente con respecto a la variable dependiente, por medio de Tau C y Tau b y $\mathrm{X}^{2}$.

\section{Consideraciones éticas}

Este proyecto se sustenta en la NOM-012-SSA3-2012, que establece los criterios para la ejecución de proyectos de investigación para la salud en seres humanos, así como en el artículo 100 de la Ley general de salud(18). Y los principios éticos de la Declaración de Helsinki. Se explicó a los participantes sobre la investigación y posteriormente quienes decidieron integrarse, lo hicieron a través de la firma de la carta de consentimiento informado. Se contó con la previa autorización para este estudio de manera escrita por los representantes del Sistema para el Desarrollo Integral de la Familia (DIF Guerrero) así como los organismos dependientes de esta.

\section{Resultados}

En la población encuestada (75 adultos mayores) predomina el sexo femenino con $52 \%$, la media de edad de 73 años. El 19\% asegura tener una mala salud y $61 \%$ la considera regular. El 52\% respondieron tener una salud igual a la del año anterior, y $31 \%$ afirma sentirse peor. Un 97,3\% no tienen una buena perspectiva de su salud, además de sentirse menos resistente a la enfermedad (Tabla 1 ).

Tabla 1. Percepción del estado de salud

\begin{tabular}{lcr}
\hline \multicolumn{1}{c}{ f } & \% \\
\hline \multicolumn{3}{l}{$\begin{array}{l}\text { Percepción del estado de salud actual en comparación } \\
\text { con el año anterior en los adultos mayores }\end{array}$} \\
Mala & 14 & 19 \\
Regular & 46 & 61 \\
Buena & 15 & 20 \\
Percepción de la salud mental actual, en comparación con \\
el año anterior en los adultos mayores & & \\
Peor & 23 & 31 \\
Igual que hace un año & 39 & 52 \\
Mucho mejor & 13 & 17 \\
\hline
\end{tabular}

De acuerdo al lugar donde vive se encontró que la mayoría de los AM considera tener una buena salud mental $(25 \%$ de la casa de día y $25 \%$ de la población libre y $23 \%$ del asilo). No obstante el $1 \%$ de los adultos mayores que están en sus domicilios tienen muy mala salud mental, 3\% de la casa de día tienen una salud mental mala, quienes consideran que su salud mental es regular son un 9\% (Asilo) 5\% (casa de día) y 4\% (población libre). Mediante $\mathrm{X}^{2}(10,003)$ no hay relación y por medio de Tau C $(0,247)$ la influencia es nula (Figura 1).

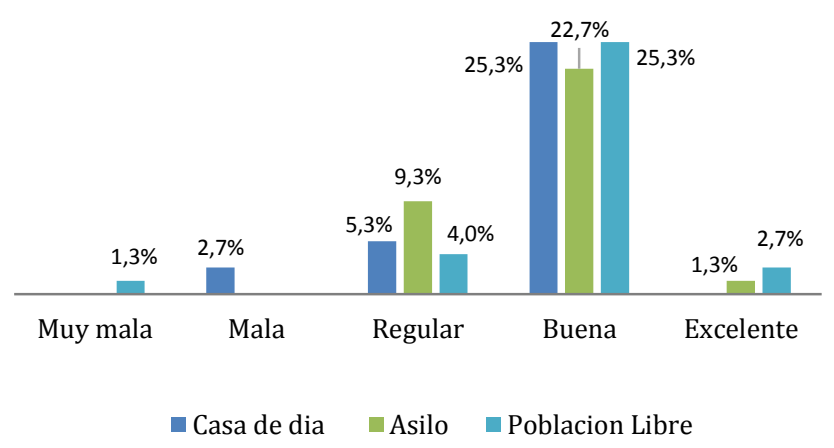

Figura 1. Salud mental de acuerdo al lugar donde vive 
Tabla 2. Aspectos de la calidad de vida

\begin{tabular}{|c|c|c|}
\hline & f & $\%$ \\
\hline \multicolumn{3}{|c|}{$\begin{array}{l}\text { Frecuencia en que sus actividades habituales han sido } \\
\text { afectadas debido a su salud física o los problemas } \\
\text { emocionales }\end{array}$} \\
\hline Siempre & 16 & 21,3 \\
\hline Casi siempre & 5 & 6,7 \\
\hline Muchas veces & 7 & 9,3 \\
\hline Algunas veces & 25 & 33,3 \\
\hline Nunca & 22 & 29,3 \\
\hline \multicolumn{3}{|c|}{$\begin{array}{l}\text { Grado de dificultad en su actividad social debido a } \\
\text { problemas físicos y emocionales }\end{array}$} \\
\hline Nada & 23 & 30,7 \\
\hline Poca & 13 & 17,3 \\
\hline Regular & 16 & 21,3 \\
\hline Bastante & 15 & 20 \\
\hline Mucho & 8 & 10,7 \\
\hline \multicolumn{3}{|c|}{ Depresión en los AM en relación a su función física } \\
\hline \multicolumn{3}{|l|}{ Sin depresión } \\
\hline Me limita poco & 1 & 1,3 \\
\hline No me limita & 1 & 1,3 \\
\hline \multicolumn{3}{|l|}{ Depresión moderada } \\
\hline Me limita poco & 25 & 33,3 \\
\hline Me limita mucho & 4 & 5,3 \\
\hline No me limita & 32 & 42,7 \\
\hline \multicolumn{3}{|l|}{ Depresión severa } \\
\hline Me limita poco & 6 & 8 \\
\hline Me limita mucho & 3 & 4 \\
\hline No me limita & 3 & 4 \\
\hline \multicolumn{3}{|c|}{ Salud mental y estados depresivos en el AM } \\
\hline \multicolumn{3}{|l|}{ Sin depresión } \\
\hline Buena salud mental & 2 & 2,7 \\
\hline \multicolumn{3}{|l|}{ Depresión moderada } \\
\hline Muy mala & 1 & 1,3 \\
\hline Mala & 2 & 2,7 \\
\hline Regular & 11 & 14,7 \\
\hline Buena & 44 & 58,7 \\
\hline Excelente & 3 & 4 \\
\hline \multicolumn{3}{|l|}{ Depresión severa } \\
\hline Regular & 3 & 4 \\
\hline Buena & 9 & 12 \\
\hline
\end{tabular}

El 71\% de los AM encuestados afirman que sus actividades habituales han sido afectadas frecuentemente debido a problemas físicos $\mathrm{y} / \mathrm{o}$ emocionales. El 52\% asegura presentar un grado de dificultad de regular a mucho en sus actividades, debido a problemas físicos y/o emocionales. La depresión en relación a la función física mediante $\mathrm{X}^{2}$ $(5,640)$ no existe relación y por medio de Tau C $(0,057)$ la influencia es nula.
La salud mental relacionada con los estados depresivos no tienen relación $\left(\mathrm{X}^{2}=2,232\right)$ y por medio de Tau C $(0,524)$ hay asociación media (Tabla 2$)$.

\section{Discusión}

En la alteración del desarrollo de actividades de la vida diaria, se encontró que el $71 \%$ de los AM se ha visto en esta situación debido a alteraciones de salud física y mental; coincidiendo con el estudio de Perú, en el que identificaron que las dimensiones más afectadas fueron las relacionadas a salud general (50\%), rol físico (61\%) y dolor corporal $(62 \%)^{(11) .}$

En Colombia una investigación realizada en el año 2013; mostró que el $71 \%$ se consideraron independientes para realizar actividades de la vida diaria, 45,7\% presentaba sintomatología depresiva y $28,3 \%$ deterioro funcional; la asociación fue negativa con: la calidad de vida, ser mujer y altos puntajes en las escalas de capacidad funcional, depresión y ansiedad(12).

La investigación de Colombia, en el año 2015 mostró que los participantes no institucionalizados presentan una mejor calidad de vida y estado mental que los institucionalizados, sin embargo, los AM institucionalizados estuvieron dentro del rango de edad de los 65 a los 85 años mientras que la otra muestra estuvo en el rango de los 55 a los 85 años de edad(13). Coincidiendo con el presente estudio, en el que la percepción que tienen los adultos mayores sobre su calidad de vida en cuanto a su estado de salud, el $19 \%$ asegura tener una mala salud y $61 \%$ considera que es regular. La mayoría de los encuestados considera tener una buena salud mental (25\% de los AM de casa de día, $25 \%$ de la población libre, y el 23\% AM del asilo). No obstante algunos AM consideran que poseen mala salud mental; así mismo no existe relación e influencia entre la salud mental de los institucionalizados y los no institucionalizados, pero se encontró asociación media entre la salud mental relacionada con los estados depresivos.

Los adultos mayores guerrerenses presentan porcentajes más altos de problemas físicos, emocionales, y sociales, que dan origen a una mala perspectiva de salud, comparado con estudios realizados en los estados de Puebla y Jalisco; donde ambos presentan una perspectiva de salud positiva, se deduce que la diferencia se radique en los determinantes económicos, sociales, culturales y 
educativos. Estos estados comparados con Guerrero tienen políticas públicas que favorecen la atención a la salud de los adultos mayores.

\section{Conclusiones}

Guerrero es uno de los estados más pobres del país, con evidente falta de políticas públicas y programas que busquen brindar una vejez plena, para la satisfacción y tranquilidad de las necesidades del adulto mayor. La calidad de vida es una prioridad dentro de las políticas públicas de las organizaciones internacionales y nacionales para retardar el deterioro funcional $\mathrm{y}$ cognitivo para un envejecimiento saludable.

Estos cambios demográficos exigen el estudio de manera continua que permita identificar las necesidades del Adulto Mayor para que los profesionales de las diferentes disciplinas mejoren la atención y por ende una mejor calidad de vida.

\section{Recomendaciones}

Explorar la calidad de vida desde un enfoque cualitativo que permita identificar los sentimientos, las emociones y las vivencias que el adulto mayor experimenta durante el proceso de envejecimiento.

Buscar el abordaje desde la perspectiva del personal de salud para conocer la sensibilidad y calidad humana en el cuidado a los adultos mayores.

\section{Agradecimientos:}

Agradecemos la apertura, disposición y apoyo de las instituciones encargadas en la atención a este grupo poblacional, a los familiares, pero sobre todo nuestro reconocimiento y admiración a los Adultos Mayores participantes por permitirnos compartir con ellos momentos inolvidables, sus experiencias de vida y el vínculo afectivo que se generó, y la enseñanza que nos transmiten para ser mejores personas y profesionales humanizados en la atención a esta población.

\section{Conflicto de intereses}

Ninguno declarado.

\section{Referencias}

1. De los Santos PV, Carmona Valdés SE. Población y Salud en Mesoamérica. Prevalencia de depresión en hombres y mujeres mayores en México y factores de riesgo. Revista Población y Salud en Mesoamérica. 2018; 15 (2):1-23.

2. Organización Mundial de la Salud. 10 datos sobre el envejecimiento. Ginebra: OMS; 2017.

3. González KD. Envejecimiento demográfico en México: análisis comparativo entre las entidades federativas. México: CONAPO; 2015.

4. Falque-Madrid L. La evidencia científica y el arte de envejecer. An Venez Nutr. 2014; 27(1):110-18.

5. Organización Mundial de la Salud. Informe mundial sobre el envejecimiento y la salud Estados Unidos de América. Ginebra: OMS; 2015.

6. Razo-González AM, Díaz-Castillo R, Morales-Rosselñ R, Cerda-Barceló R. Metaanálisis del concepto de calidad de vida en América Latina. Una nueva propuesta: sentido de vida. Revista CONAMED. 2014; 19 (4):149-156.

7. Rubio-Olivares DY, Rivera-Martínez L, Borges-Oquendo L, González-Crespo FV. Calidad de vida en el Adulto Mayor. Varona. 2015;(61):1-7.

8. Rodríguez. P. La atención integral centrada en la persona. Madrid: Fundación Pilares; 2010.

9. Cano-Ramírez CZ, García-Isaac DA, García-Gutiérrez RA Gloria-Marrufo SY. Vejez generativa: construyendo puentes entre la empresa y el adulto mayor. Vincula Tégica. 2016; 2(1):932-55.

10. Vera M. Significado de la calidad de vida del adulto mayor para sí mismo y para su familia. An Fac Med Lima. 2007; 68 (3):284 -290.

11. Chafio-Prada PM, Gallo-Torres GG. Calidad de vida del Adulto Mayor con Diabetes Mellitus que sea tiende en el Centro De Salud José Olaya Chiclayo - 2015. [Tesis]. Chiclayo: Universidad Señor de Sipán, Facultad de ciencias de la Salud; 2016 Enero 27

12. Estrada A, Cardona D, Segura AM, Chavarriaga LM, Ordoñez J, Osorio JJ. Calidad de vida de los adultos mayores de Medellín. 2011; 31(4):492-502.

13. Bedoya-Jácome MF. Calidad de vida en adultos mayores, ancianos jóvenes y ancianos maduros institucionalizados y no institucionalizados en la ciudad de Pereira [Tesis]. Pereira: Universidad Católica de Pereira; 2015.

14. Silva Ortiz N. Calidad de vida de los integrantes de la Asociación Adulto Mayor La Tulpuna. Cajamarca, 2016 [Tesis]. Cajamarca: Universidad Nacional de Cajamarca; 2017.

15. Acosta-Quiroz CO, Tánori_Quintana J, García R, Echeverria S, Vales-García JJ, Rubio L. Soledad, depresión y calidad de vida en adultos mayores mexicanos. Psicología y Salud. 2017; 27 (2):179-88.

16. González-Bernal J, de la Fuente-Anuncibay R. Desarrollo humano en la vejez: un envejecimiento optimo desde los cuatro componentes del ser humano. International Journal of Developmental and Educational Psychology. 2014; 7(1):121129.

17. Institut Municipal d'Investigació Mèdica. Cuestionario de Salud SF-36 (versión 2). Barcelona: IMAS; 2003.

18. Secretaria de Salud. NORMA Oficial Mexicana NOM-012SSA3-2012, Que establece los criterios para la ejecución de proyectos de investigación para la salud en seres humanos. México: DOF; 2013. 